\title{
Synthesis and study of the reactivity of 1,1'-oxalyl di(2-methylimidazole) in reactions with $N$-nucleophiles
}

\author{
(C) Pyotr P. Purygin,* Viktoriya D. Fedotova, \\ Vitaly Yu. Alekseev, and Yury P. Zarubin ${ }^{+}$ \\ Department of Inorganic Chemistry. Samara National Research University. Moskovskoye Shosse, 34. \\ Samara, 443086. Samara Region. Russia. Phone: +7 (846) 334-54-59.E-mail: puryginpp2002@mail.ru
}

\begin{abstract}
*Supervising author; ${ }^{+}$Corresponding author
Keywords: 1,1'-oxalyl di(2-methylimidazole), synthesis, reactivity, nucleophilic susceptibility, electrophilic susceptibility, calculations, molecular-mechanical, quantum-chemical, thermodynamic parameters, biological activity, prediction.
\end{abstract}

\section{Abstract}

This article describes the synthesis of 1,1'-oxalyl di(2-methylimidazole), which was obtained in two stages. At the first stage of the synthesis, 1-trimethylsilyl-2-methylimidazole and oxalyl chloride were obtained. At the second stage of the synthesis, the target product was obtained by reacting 1-trimethylsilyl-2methylimidazole with oxalyl chloride. The product was a dark crystalline substance, the yield of the final product was $83 \%$. The structure of 1,1'-oxalyl di(2-methylimidazole) was confirmed by IR and ${ }^{1} \mathrm{H}$ NMR spectroscopy. In the IR spectra, characteristic absorption bands were found, confirming the presence of the corresponding functional groups in the structure of the compound, in the ${ }^{1} \mathrm{H}$ NMR spectrum, proton signals with characteristic chemical shifts for the corresponding functional groups were found. The PASS online software predicted the types of biological activity of the compound under study. The most probable possibilities of application in the treatment of urological diseases, diabetes mellitus, staphylococcal, oncological diseases, antiaggregatory action. For the 1,1'-oxalyl di(2-methylimidazole) molecule, 6 conformers were found, which were three pairs of enantioconformers in the Molecular Operating Environment 2009.10 software, for which the electrostatic potential, nucleophilic and electrophilic susceptibility surfaces were calculated in the SCIGRESS Modeling 3.1.4 software. It was shown that the interaction with nucleophilic reagents is most typical for the 1,1'-oxalyl di(2-methylimidazole) molecule. Calculations of the potential energy of the conformers of 1,1'-oxalyl di(2-methylimidazole) molecules have been carried out. Using 1,1'-oxalyl di(2-methylimidazole), the syntheses of acetanilide, benzanilide and diethylformamide were carried out. These syntheses were carried out in order to test the reactivity and the possibility of amide-type bond formation upon activation of the carboxyl group in carboxylic acids. The reactions were carried out in one stage without isolation of the intermediate 2-methylimidazolide of the corresponding carboxylic acid. In this case, the proposed reaction mechanism should be two-stage. Possible structures of reactants, transition states, and reaction products in the interaction of 1,1'-oxalyl di(2-methylimidazole) with carboxylic acids and amines have been calculated by methods of computational chemistry.

\section{References}

[1] V.I. Ivansky. Chemistry of heterocyclic compounds. Moscow: Vysshaya shkola. 1978. 559p. (russian)

[2] V.A. Kataev. Thietanes based on benzimidazole and imidazole. Synthesis, structure and biological properties: abstr. diss. ... doct. farm. sciences. Moscow. 2006. 46p. (russian)

[3] L. Birkofer, P. Richer, A. Ritter. Chem. Ber. 1960. Vol.93. P.2804-2809.

[4] D.A. Filimonov, A.A. Lagunin, T.A. Gloriozova, A.V. Rudik, D.S. Druzhilovskii, P.V. Pogodin, V.V. Poroikov. Chemistry of Heterocyclic Compounds. 2014. Vol.50. No.3. P. 444-457. DOI:10.1007/s10593014-1496-1

[5] P.C. Chan, R.C. Sills, G.E. Kissling, A.F. Nyska, W.T. Richter. J. Arch. Toxicol. 2008. Vol.82. P.399-411.

[6] D.A. Kirichenko. Siberian medical journal. 2007. Vol.4. No.6. P.13-15. (russian)

[7] A.V. Smolyakova. Pharmaceutical Bulletin. 2010. Vol.2. No.6. P.21-32. (russian) 